\title{
High Purity Synthesis of Carbon Nanotubes by Methane Decomposition Using an Arc-Jet Plasma
}

\author{
S. I. Choi*, J. S. Nam, C. M. Lee, S. S. Choi, J. I. Kim, J. M. Park and S. H. Hong ${ }^{* *}$ \\ Department of Nuclear Engineering, Seoul National University \\ Seoul 151-742, Korea
}

\begin{abstract}
High purity carbon nanotubes are synthesized by methane decomposition using an arc-jet plasma of high temperature $(5000-20000 \mathrm{~K})$. Since the arc-jet plasma process is continuous and easily scalable, it is a promising technique for the large-scale commercial production of carbon nanotubes. In this experimental work, the arc-jet plasma is generated by a dc non-transferred plasma torch, in which a mixture of argon and hydrogen is used as a plasma forming gas and nickel powder as a metal precursor. Morphology, crystallization degree and purity of the carbon nanotubes in the soot produced under various processing conditions are evaluated by using scanning electron microscopy, transmission electron microscopy, Raman spectroscopy and thermogravimetric analysis. From the results of these material analyses, we have found that multi-walled carbon nanotubes of high purity were produced in the optimal operating condition. In addition, the thermal plasma characteristics for the carbon nanotubes growth are discussed from numerical simulation result of the arc-jet plasma.
\end{abstract}

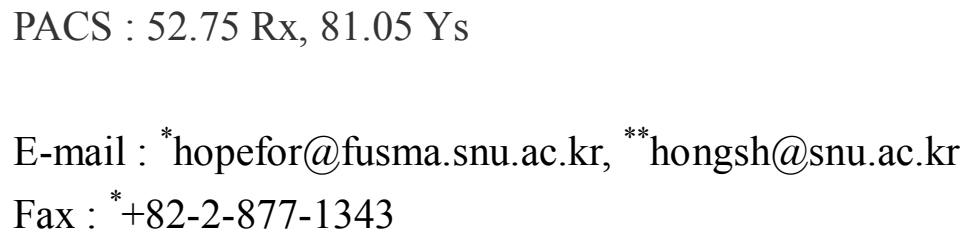

Keywords: carbon nanotubes, arc-jet plasma, thermal plasma, decomposition of methane, synthesis 


\section{INTRODUCTION}

Carbon nanotubes (CNTs) are regarded as a powerful material for various applications in the nano-technology field because of their unique properties. An arc discharge method has been widely used for the CNTs synthesis since it can produce highly crystallized CNTs by high temperature ambient (about $5000 \mathrm{~K}$ ) [1,2]. However, it is still difficult to adapt the conventional arc discharge method to commercial use because of its drawbacks of poor purity synthesis and non-continuous process. Especially the non-continuous process stems from the limited lifetime of the consumable graphite electrode used as a carbon source and the deposit of the product on the reactor inner wall. In other words, the non-continuous synthesis process has to have a shutdown period of operations to reload a new graphite electrode and collect the product in a vacuum destruction phase.

Recently we have reported a continuous synthesis technique of CNTs without a process shutdown by decomposition of methane $\left(\mathrm{CH}_{4}\right)$ using an arc-jet plasma [3]. In this technique, CNTs are produced in a floating condition by introducing $\mathrm{CH}_{4}$ and metal precursor into the arc-jet flame, which has thermal plasma properties of high temperature $(5000-20000 \mathrm{~K})$ and high velocity $(100-1000 \mathrm{~m} / \mathrm{s})[4,5] . \mathrm{CH}_{4}$ is directly converted into solid carbon containing CNTs in the hot plasma zone by the following decomposition reaction:

$$
\mathrm{CH}_{4} \text { (gas) } \rightarrow \mathrm{C} \text { (solid) }+2 \mathrm{H}_{2} \text { (gas) }
$$

Hydrogen as a new synthetic fuel is additionally obtained in this process.

In this work, the arc-jet plasma process is optimized for high purity synthesis of CNTs. We present the effects of processing conditions, such as torch nozzle diameter, hydrogen gas mixing and $\mathrm{CH}_{4}$ flow rate, on the synthesis of CNTs using this arc-jet plasma method. The material analyses of the soot obtained under the various process conditions are performed by using scanning electron microscopy (SEM), transmission electron microscopy (TEM), Raman spectroscopy and thermogravimetric analysis (TGA) to investigate morphology, crystallization degree and purity of the CNTs in the soot. The thermal plasma characteristics for the growth of CNTs are discussed from numerical simulation results of the arc-jet plasma.

\section{EXPERIMENAL}


The experiment system for the CNTs synthesis mainly consists of a dc plasma torch, injectors of reactant gas and catalyst and a collection tube. A schematic diagram of the system is shown in Fig. 1. The arc-jet plasma is generated by the dc plasma torch, which has a cathode of $12-\mathrm{mm}$ diameter and $60^{\circ}$ conical angle, and an anode of 7-mm or 9-mm inner diameter. Pure argon or a mixture of argon and hydrogen is used as a plasma gas. Argon flow rate is fixed with $25 \mathrm{slpm}$ and hydrogen flow rate is varied in the range of 0 $3 \mathrm{slpm} . \mathrm{CH}_{4}$ is used as a carbon source, and nickel powder of about 3- $\mu \mathrm{m}$ diameter is used as a metal precursor. $\mathrm{CH}_{4}$ of $1-4 \mathrm{slpm}$ and nickel powder of $0.44 \mathrm{~g} / \mathrm{min}$ are introduced at the anode exit. For sufficient mixing of precursors with the arc-jet plasma, stainless injection tubes of 1.5- $\mathrm{mm}$ inner diameter are used, and their injecting directions are oriented at $45^{\circ}$ to the axis of the plasma jet toward the upstream direction [6]. The arc-jet plasma is generated with a dc current of $350 \mathrm{~A}$, while the ambient pressure is maintained at atmosphere condition.

The CNT samples produced in the experiment are collected by a stainless steel collection tube for their material analyses and tests by using field emission SEM (JEOL, JSM-6330F), high resolution TEM (JEOL 3011), TGA (TA Instruments, TGA2050) and Raman spectrometer (Jobin-Yvon/Spectra-Physics, T64000). The SEM and TEM are operated at 10 and $300 \mathrm{kV}$, respectively. Approximately $8 \mathrm{mg}$ of the produced soot is used for TGA in the measurement conditions with $10{ }^{\circ} \mathrm{C} / \mathrm{min}$ ramp rate from room temperature to $800{ }^{\circ} \mathrm{C}$ at an air flow rate of $100 \mathrm{sccm}$.

\section{RESULTS AND DISCUSSION}

A two-dimensional numerical simulation for the arc-jet plasma used for the synthesis is conducted in order to understand the thermal flow characteristics of arc-jet thermal plasma and investigate the effects of operating conditions on the characteristics. This simulation is based on a thermal plasma model used generally for description of the arcjet plasma [7,8]. Figure 2 shows the calculated results for the typical temperature and velocity distributions of the arc-jet plasma. It is seen that the arc-jet flame with high temperature of about $10000 \mathrm{~K}$ and high velocity of about $300 \mathrm{~m} / \mathrm{s}$ is blown out at the torch exit. Both of the plasma temperature and velocity rapidly decrease with increasing the distance in the axial direction.

The effects of anode diameter and hydrogen mixing for forming an argon-hydrogen mixture plasma on the plasma characteristics are shown in Fig. 3. From the simulation 
results, it is found that the hydrogen gas mixing and the anode diameter play an important role in the profile control of plasma temperature and velocity. When 1-slpm hydrogen gas is mixed with argon plasma gas, the high temperature region of the jet flow is noticeably expanded toward the axial direction compared with no hydrogen mixing. The plasma velocity increases a little when hydrogen gas is added. On the other hand, in the case of larger diameter anode of $9 \mathrm{~mm}$, the plasma velocity considerably reduces from that of the case of 7-mm anode diameter, but the temperature dose not decrease appreciably.

Some raw soot is obtained from the collection tube after the synthesis process, and the CNTs are eventually found in the soot through its material analyses, which are conducted without a purification process.

Figure 4 shows SEM images of the raw-soot produced with 2-slpm $\mathrm{CH}_{4}$ and 9-mm inner diameter of the anode at the different $\mathrm{H}_{2}$ flow rates of 0,1 , and $3 \mathrm{slpm}$, respectively. As seen in Fig. 4(a), CNTs are hardly found in the case that hydrogen is not mixed as a part of plasma gas. However, in Fig. 4(b), lots of CNTs are produced when 1-slpm hydrogen is mixed with argon. In the latter case, it is measured that the arc voltage is increased by 1.5 times compared with the former case at the same arc current of $350 \mathrm{~A}$, which means that the input electric power into the arc-jet plasma accordingly increases. As shown previously in the numerical results, the hydrogen gas mixing largely elevates the plasma temperature. In addition, the hydrogen gas mixing increases thermal conductivity of the high temperature gas. When hydrogen is not mixed with argon, catalytic nickel particles of large size are observed in the SEM image. But, when 1 -slpm hydrogen is mixed with argon, they are not found in the image. Therefore, it is concluded from this observation that a high-temperature and high-enthalpy plasma produced by addition of hydrogen is required for CNTs production since in a pure argon plasma, the catalytic nickel powder does not evaporate sufficiently to form nano-sized metal particles, which is essential for the growth of CNTs.

But, in Fig. 4(c) for 3-slpm hydrogen, the fraction of CNTs in the soot is reduced again when an excessive hydrogen gas is mixed. In this case, the arc-jet plasma is unstable and the CNTs growth appears to be disturbed by plasma instability caused by the excessive hydrogen mixing. It is well known that a hydrogen gas mixing in the nontransferred-type plasma torch causes an arc fluctuation by re-strike motion inner the 
nozzle [9]. This result indicates that the plasma stability plays an important role in the high purity synthesis of CNTs.

Figure 5 shows SEM images of the sample produced with 1-slpm hydrogen and 9$\mathrm{mm}$ anode diameter at the different $\mathrm{CH}_{4}$ flow rates of 1 slpm and 4 slpm. The experimental result for 2-slpm $\mathrm{CH}_{4}$ is given previously in Fig 4. Since $\mathrm{CH}_{4}$ is used in the present experiment as a carbon source for converting to solid carbon, the flow rate of $\mathrm{CH}_{4}$ is expected to determine the production rate of solid carbon material. However, it is shown in Figs. 4 and 5 that the flow rate of $\mathrm{CH}_{4}$ also has an influence on purity of CNTs in the soot. When the flow rate of $\mathrm{CH}_{4}$ is so low as 1-2 slpm, a considerable amount of CNTs are produced as seen in Fig. 4(b) and Fig. 5(a). However, for a high flow rate of 4-slpm $\mathrm{CH}_{4}, \mathrm{CNTs}$ are hardly found as observed in Fig. 5(b), where nickel particles of large size are instead observed. Since an excessive flow rate of $\mathrm{CH}_{4}$ lowers the plasma temperature and enthalpy, the nickel powder appears not to be evaporated sufficiently. From the above experimental results, it is important for the CNTs synthesis by decomposition of $\mathrm{CH}_{4}$ to provide the sufficiently high temperature and enthalpy of the plasma using the arc-jet plasma torch during the synthesis process.

Figure 6 shows the SEM image of a sample produced in the condition with 7-mm anode inner diameter, 1-slpm hydrogen and 2-slpm $\mathrm{CH}_{4}$. It is observed that the fraction of CNTs in the soot becomes lower than in the case of 9-mm anode diameter. It is noticed in Fig. 3 for the numerical simulation that the 7-mm anode diameter raises the plasma velocity. Hence, the plasma velocity seems to affect the CNTs growth in the synthesis process using the arc-jet plasma. Because an enough reaction time is required for the growth of CNTs during their axial flight along the jet flame, it is considered that lower plasma jet velocity enhances the CNTs production

Figure 7 shows typical TEM images of the soot produced by the arc-jet plasma method for the case of 1-3 slpm hydrogen, 9-mm anode diameter and 2-slpm $\mathrm{CH}_{4}$. In Fig. 7(a), a number of CNTs are seen along with other particles. The produced CNTs are estimated to have diameters of 30-60 nm and lengths of longer than $1 \mu \mathrm{m}$. The exact length could not be measured because no CNTs were observed with both ends of CNTs appeared at the same time. And it is frequently observed that metal particles are filled not in the ends of the tubes, but in the middle. CNTs of small diameter are sometimes observed as shown in Fig. 7(b). From the high magnification image for the raw soot, we have found that the CNTs characterized by hollow inner space were obviously produced 
in our method.

Figure 8 presents a TGA graph of the raw-soot, which contains the most CNTs produced with the processing condition of 2-slpm hydrogen, 9-mm anode diameter and 1-slpm $\mathrm{CH}_{4}$. In this figure, the bold and dash lines correspond to TG and DTG, respectively. This graph indicates that a residual weight of the soot is about 0.82 , which is contributed mainly from nickel particles. This implies that the conversion efficient of $\mathrm{CH}_{4}$ into the solid carbon soot is still not high.

It is also observed from this figure that an initial burning temperature of the raw-soot is about $480{ }^{\circ} \mathrm{C}$ and that there is nearly one stepwise weight loss which can be assigned to multi-wall CNTs. Conventionally, the other DTG peak assigned to amorphous carbon is observed around $400{ }^{\circ} \mathrm{C}$. DTG is known as a common method used for quantitative analysis, and the content of each species can be determined from its peak area in DTG. Thus the yield of CNTs in the optimal processing condition is estimated as over $90 \%$ in the soot produced by this arc-jet plasma method. This indicates that the high purity synthesis of CNTs is achievable by decomposition of $\mathrm{CH}_{4}$ using the arc-jet plasma.

Figure 9 is the typical Raman spectra of the raw-soot produced under the condition of 1-slpm hydrogen and 1- slpm methane. This spectral curve is recorded at room temperature using an excitation wavelength of $514.5 \mathrm{~nm}$. In the high-frequency range from 1200 to $1700 \mathrm{~cm}^{-1}$, two peaks are observed at 1354 and $1583 \mathrm{~cm}^{-1}$. A peak at 1354 $\mathrm{cm}^{-1}$ corresponds to a disorder-induced phonon mode (D-band), and a peak at $1583 \mathrm{~cm}^{-1}$ can be assigned to a Raman-allowed phonon mode (G-band). It is known that the intensity ratio, G/D, indicates the crystallization degree of grown CNTs. Figure 9 shows that the intensity of $\mathrm{G}$ band is a bit higher than that of D band. Generally, it is known that the ratio is very high in the arc discharge method, while low in the catalytic pyrolysis method. According to Fig. 9 for the present arc-jet plasma synthesis, the crystallization degree of CNTs is low compared with that by the conventional arc discharge method. Although our method is regarded as a high temperature process like the arc discharge, the crystallization degree of the CNTs produced here is unexpectedly low. The reason of this poor crystallization is attributed to the CNTs growth in a low temperature region below $2000 \mathrm{~K}$, because the temperature and velocity of the arc-jet plasma rapidly decrease along the axial direction at the torch exit as seen in the numerical simulation of Fig. 2. Therefore, further intensive research is required for increasing the crystallization degree of grown CNTs. 


\section{CONCULUSION}

For high purity production of CNTs in this experimental work, we have optimized the synthesis process by decomposition of $\mathrm{CH}_{4}$ using the arc-jet plasma. From the experimental study performed under the various operating conditions, it has been found that the fraction of CNTs in the soot considerably increases by mixing of hydrogen properly with argon as a mixture plasma gas, and that the excessive flow rate of $\mathrm{CH}_{4}$ reduces the purity of CNTs in the soot. Also, CNTs are more abundantly produced in the condition of larger inner diameter of the anode. By the numerical investigations on the effects of operating conditions on the thermal flow characteristics of the arc-jet plasma, higher plasma temperature and lower jet velocity enhance the CNTs growth in the arcjet plasma method.

It has been observed from the TEM images that CNTs are produced typically with diameters of 30-60 nm and lengths of longer than $1 \mu \mathrm{m}$. The high purity CNTs produced by this method in the optimal condition have the weight purity of over $90 \%$ and the crystallization degree still lower than that of the conventional arc discharge method.

\section{ACKNOWLEDGMENT}

This work was supported by Korea Institute of Science and Technology Evaluation and Planning (KISTEP) of the Ministry of Science and Technology in Korea 


\section{REFERENCES}

[1] D. Qian, E.C. Dickey, R. Andrews and T. Rantell, Appl. Phys. Lett. 76, 2868 (2000)

[2] S. Cui, P. Scharff, C. Siegmund, D. Schneider, K. Risch, S. Klötzer, L. Spiess, H. Romanus and J. Schawohl, Carbon 42, 931 (2004)

[3] S. I. Choi, J. S. Nam, J. I. Kim, T. H. Hwang, J. H. Seo, and S. H. Hong, to be published in Thin Solid Film (2005)

[4] Maher I. Boulos, IEEE T. Plasma Sci. 19, 1078 (1991)

[5] Sooseok Choi, Tae Hyung Hwang, Jun Ho Seo, Dong Uk Kim, and Sang Hee Hong, IEEE Trans. Plasma Sci. 32, 473 (2004)

[6] Gervais Soucy, Jerzy W. Jurewicz, and Maher I. Boulos, Plasma Chem. Plasma Process. 14, 59 (1994)

[7] D. A. Scott, P. Kovitya, and G. N. Haddad, J. Appl. Phys. 66, 5232 (1989)

[8] K. D. Kang and S. H. Hong, IEEE Trans. Plasma Sci. 24, 89 (1996)

[9] M. P. Collares, E. Pfender, IEEE Trans. Plasma Sci. 25, 864 (1997) 


\section{FIGURE CAPTIONS}

Figure 1. Schematic of an arc-jet plasma reactor used for carbon nanotubes synthesis by decomposition of $\mathrm{CH}_{4}$

Figure 2. The temperature and velocity distributions of arc-jet plasma calculated by a simulation (arc current: $350 \mathrm{~A}, \mathrm{Ar}: 25 \mathrm{slpm}, \mathrm{H}_{2}$ : 1 slpm, anode diameter: $9 \mathrm{~mm}$ )

Figure 3. Plasma characteristic profiles along the arc centerline depending on anode diameter and hydrogen gas mixing; (a) temperature and (b) velocity (arc current: $350 \mathrm{~A}$, Ar: $25 \mathrm{slpm})$

Figure 4. SEM images of CNTs produced with the different flow rates of $\mathrm{H}_{2}$ : (a) 0 slpm, (b) 1 slpm, and (c) 3 slpm

Figure 5. SEM images of CNTs produced with the different flow rates of $\mathrm{CH}_{4}$ : (a) 1 slpm and (b) 4 slpm

Figure 6. SEM images of CNTs produced with an anode of 7-mm inner diameter $\left(\mathrm{H}_{2}: 1\right.$ slpm, $\mathrm{CH}_{4}: 2$ slpm)

Figure 7. Typical TEM images of the raw-soot produced by decomposition of $\mathrm{CH}_{4}$ in the arc-jet plasma: (a) 1-slpm $\mathrm{H}_{2}$ and (b) 3-slpm $\mathrm{H}_{2}$

Figure 8. TGA curves of the raw-soot produced by decomposition of $\mathrm{CH}_{4}$ in the arc-jet plasma in the condition of 2-slpm $\mathrm{H}_{2}$ and 1-slpm $\mathrm{CH}_{4}$

Figure 9. Typical Raman spectra of the raw-soot produced by decomposition of methane in the arc-jet plasma $\left(\mathrm{H}_{2}: 1 \mathrm{slpm}, \mathrm{CH}_{4}: 1 \mathrm{slpm}\right)$ 


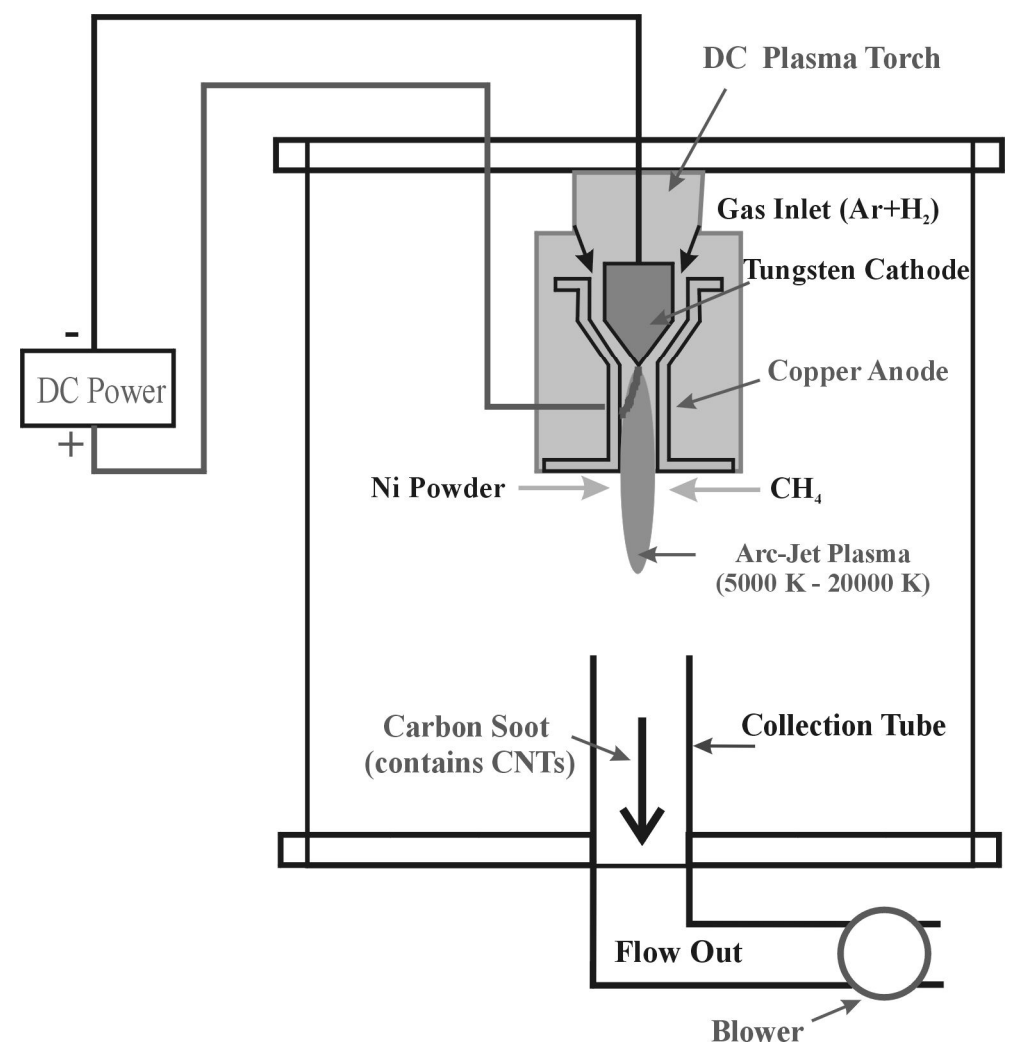

Fig. 1 


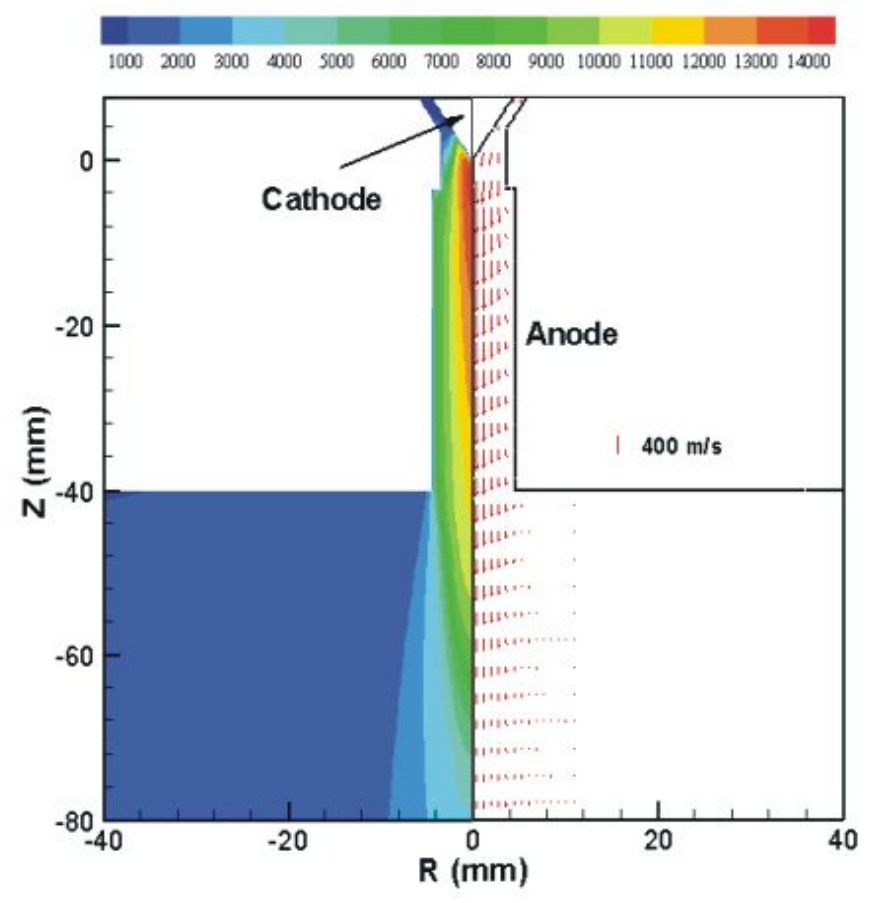

Fig. 2 

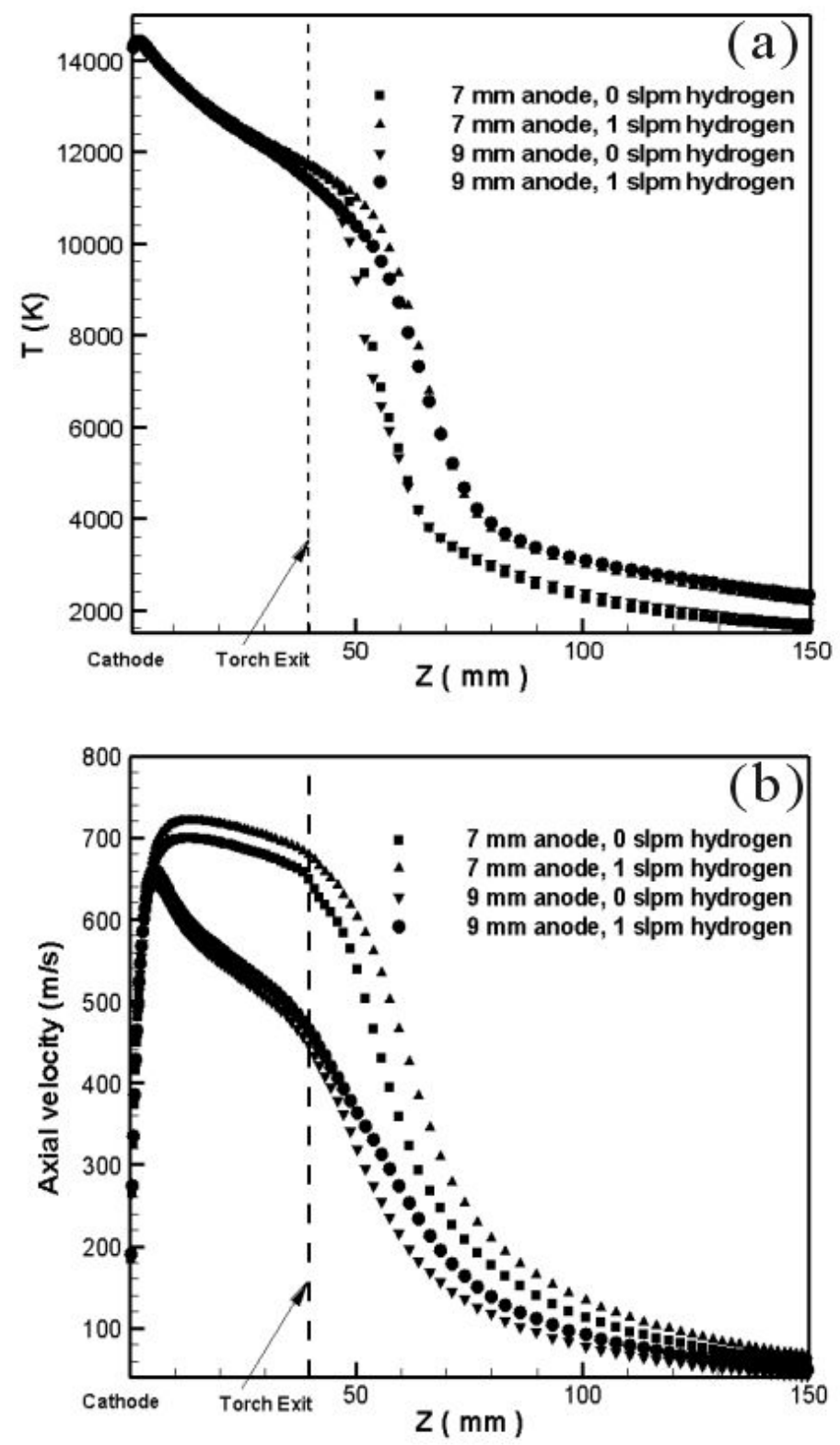

Fig. 3 


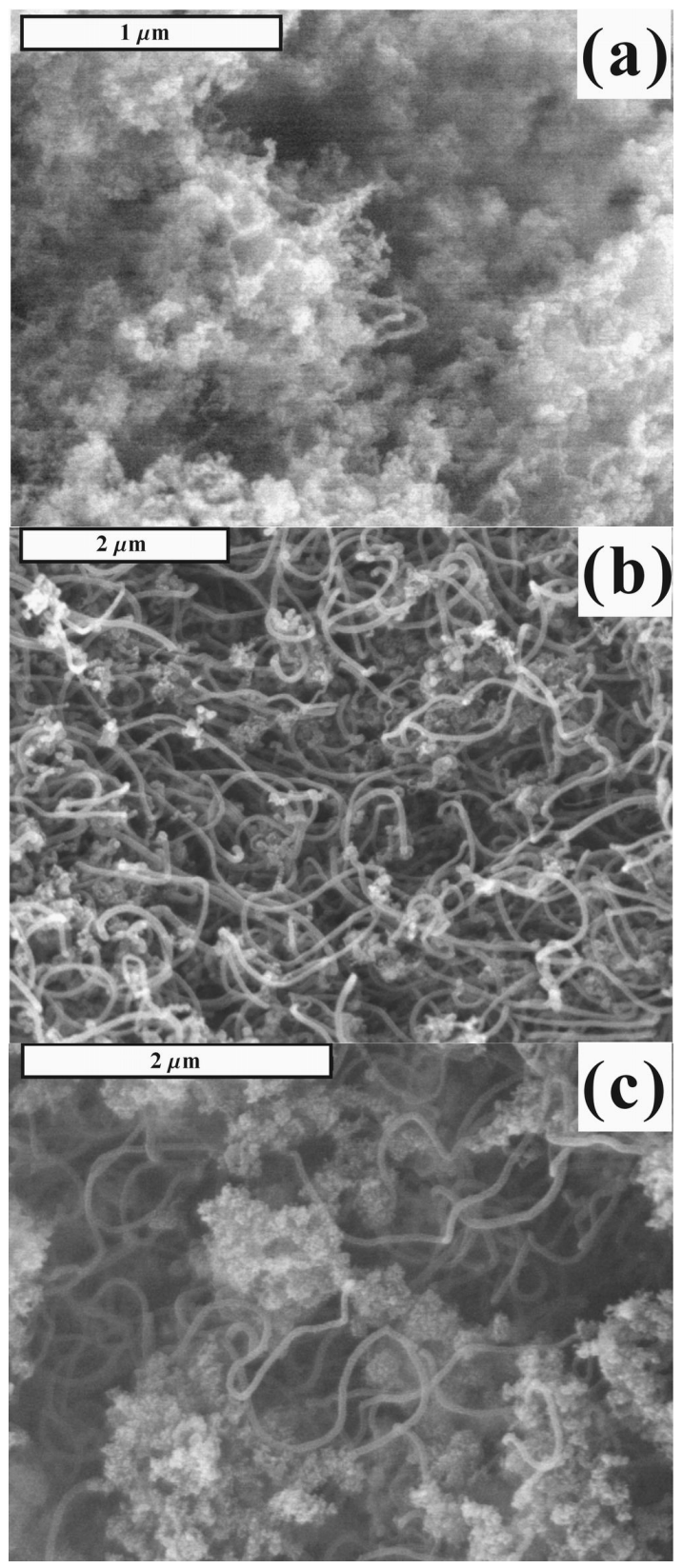

Fig. 4 


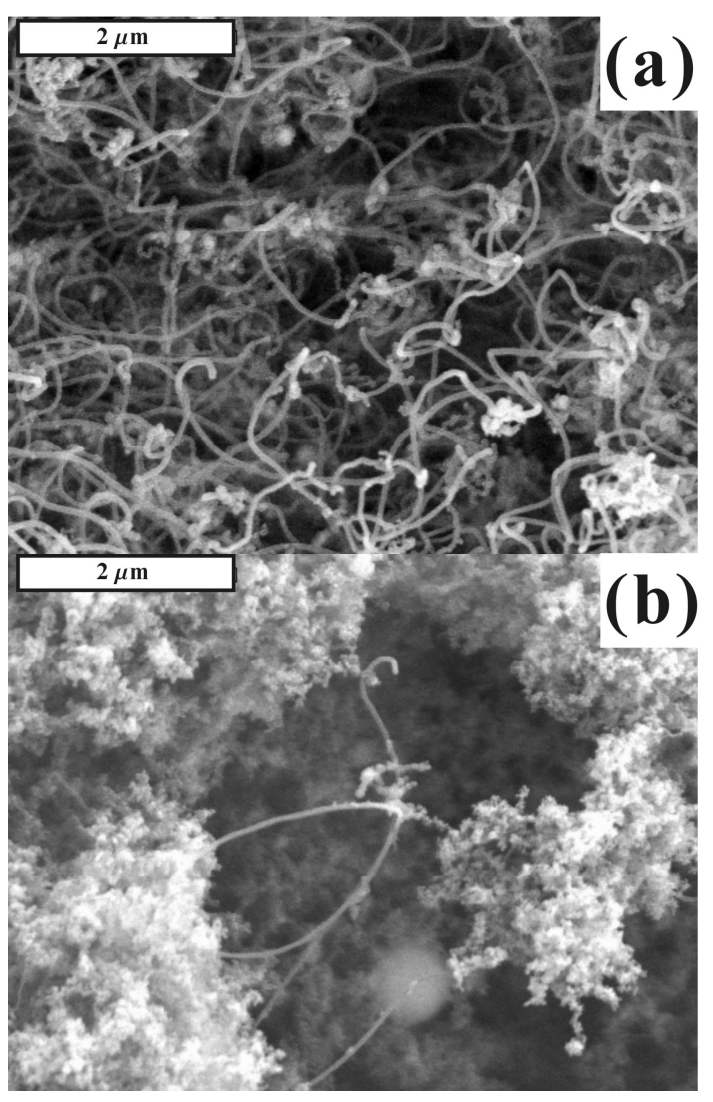

Fig. 5 


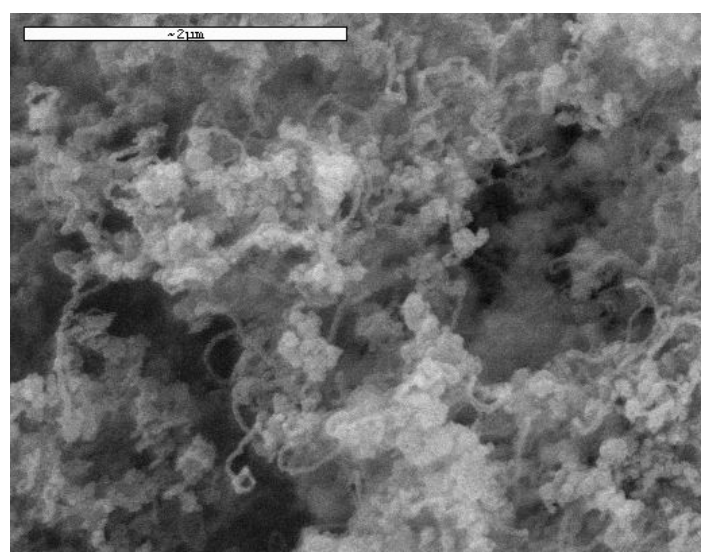

Fig. 6 


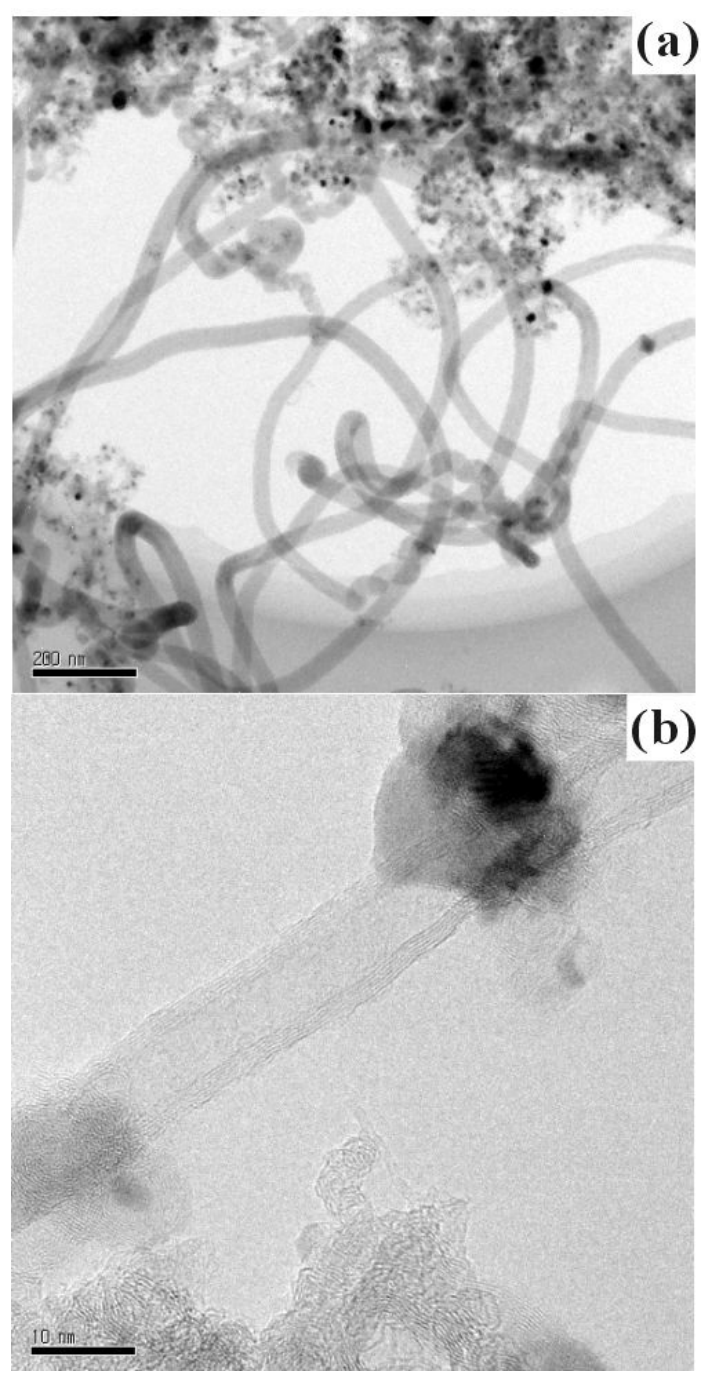

Fig. 7 


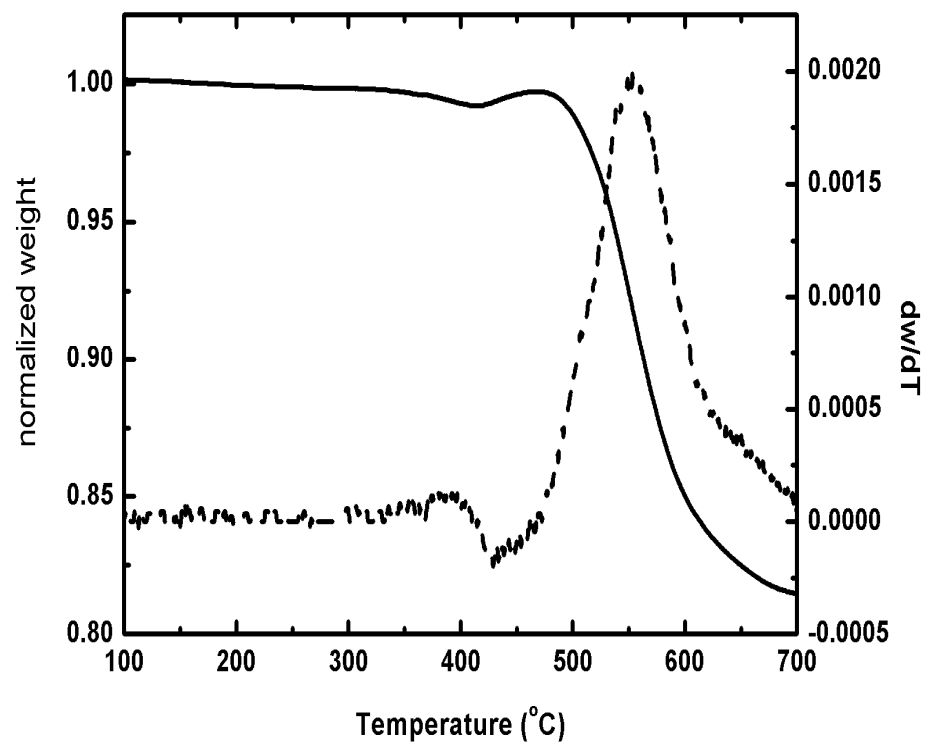

Fig. 8 


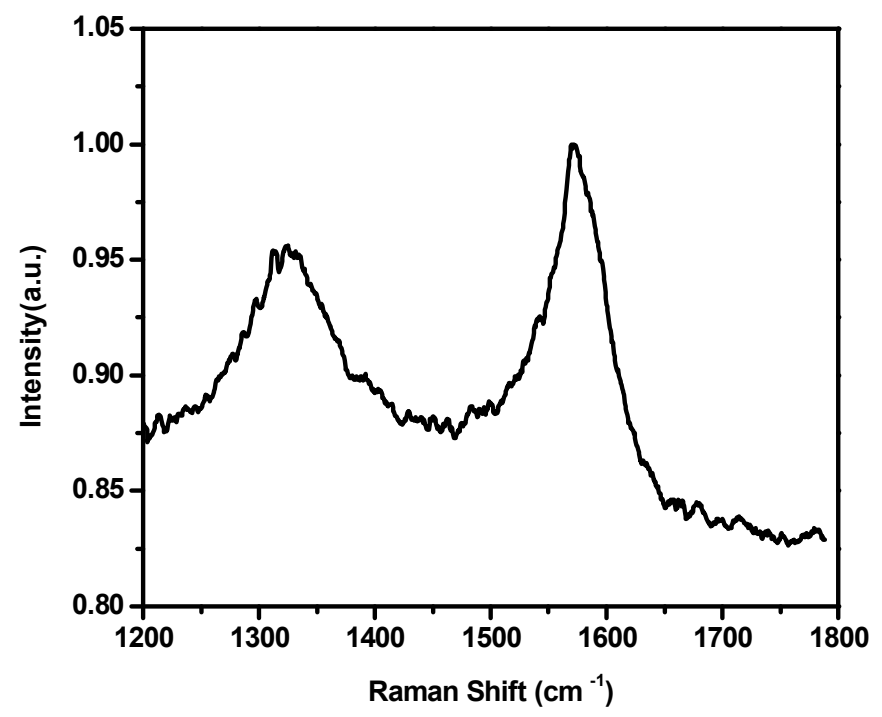

Fig. 9 